\title{
Neural Network Architecture for Cognitive Navigation in Dynamic Environments
}

\author{
José Antonio Villacorta-Atienza and Valeri A. Makarov
}

\begin{abstract}
Navigation in time-evolving environments with moving targets and obstacles requires cognitive abilities widely demonstrated by even simplest animals. Nevertheless, it is a long-standing challenging problem for artificial agents. Cognitive autonomous robots coping with this problem must solve two essential tasks: i) understand the environment in terms of "what may happen" and "how I can deal with this", and ii) learn successful experiences for their further use in an automatic subconscious way. The recently introduced concept of Compact Internal Representation (CIR) provides the ground for both tasks. CIR is a specific cognitive map, which compacts time-evolving situations into static structures containing information necessary for navigation. It belongs to the class of global approaches, i.e. it finds trajectories to a target when they exist but also detects situations when no solution can be found. Here we extend the concept on situations with mobile targets. Then using CIR as a core we propose a closed-loop neural network architecture consisting of "conscious" and "subconscious" pathways for efficient decisionmaking. The conscious pathway provides solutions to novel situations if the default subconscious pathway fails to guide the agent to a target. Employing experiments with roving robots and numerical simulations we show that the proposed architecture provides the robot with cognitive abilities and enables reliable and flexible navigation in realistic time-evolving environments. We prove that the subconscious pathway is robust against uncertainty in the sensory information. Thus if a novel situation is similar but not identical to the previous experience (due to e.g. noisy perception) then the subconscious pathway is able to provide an effective solution.
\end{abstract}

Index Terms-Neural Networks, Dynamical Systems, Cognition, Internal Representation, Cognitive maps

\section{INTRODUCTION}

$\mathbf{T}$ HE main advantage of animate mobile creatures with respect to plants is the possibility to interact actively with the environment. This postulates time as a vital dimension. The agent best dealing with this "extra" dimension receives an evolutionary advantage. Even simplest animals exhibit surprisingly efficient behaviors in complex time-evolving environments (see e.g. [1], [2]). It has been argued that the global brain function is geared towards the implementation of intelligent motricity (see [3] for review). Therefore efforts in creating artificial agents possessing cognitive abilities should involve solutions found by Nature.

There exists growing experimental evidence that cognitive motor skills developed by higher mammals are based on effective Internal Representation (IR) of the external world and the body [4]-[7]. IR makes possible mental simulations, goal planning, testing alternative behaviors and, as a consequence, an intelligent decision-making [8]. However, the mechanisms

J.A. Villacorta-Atienza and V.A. Makarov are with the Department of Applied Mathematics, Complutense University of Madrid, Av. Complutense s/n, 28040, Madrid, Spain (e-mail: vmakarov@ mat.ucm.es). behind IR are barely understood both from theoretical and experimental viewpoints [9], [10].

In general IRs must be simple enough to enable their efficient learning, storage in memory, classification, and retrieval. Perception of the environment implies concurrence and confluence of diverse sensory modalities. Then the received complex information about the external world must be properly reduced and structured to create useful IR. Thus two main questions arise:

- What information is vital for motor behavior?

- How can it be structured and stored in a neural network?

IR of static situations (all objects in the arena are immobile) can be thought about as an abstract "copy" of the external world. For the purpose of navigation an agent can just project static obstacles and targets within the visual area into a mental map and then plan a route to a target. The concept of Artificial Potential Field (APF) provides an elegant and mathematically simple implementation for this task (see [11] for review). Khatib in his pioneering works (see [12] and references therein) describes the philosophy of APF as follows. The robot moves in a field of forces. The target is an attractive pole while obstacles are repulsive surfaces. Thus targets and obstacles are mapped into two-dimensional network of locally coupled neurons as sources and sinks, respectively. Then the diffusion process generates a potential field with maxima and minima at the targets' and obstacles' positions, respectively. The agent can reach a target by "climbing" against the gradient of this potential field.

IR of dynamic situations (objects can move in the arena) demands higher-level cognitive abilities. Generalizing the static approach, one could generate a sequence of static IRs made for each time instant like frames in a movie. The APF approach has been also extended to account for such quasi-static solutions [12]. Then different approaches were proposed: recalculating the whole potential field each time a change happens [13], or updating the boundary conditions related to the moving obstacles [14]. This, however, has a number of internal pitfalls: from the obvious increase of the response time and virtually infinite memory capacity required for learning dynamic situations, to the ambiguous dynamic treatment of essentially static information. Another drawback is the problem of local minima and inefficient path planning [15].

Recently several alternative solutions to the IR problem have been proposed (see e.g. [16], [17]). The derived navigational rules imitate behaviors of insects and other simple organisms. In particular, they explore random or chaotic search for a target [18], path-integration to return home [19], recognitiontriggered response, maximization of the probability of infor- 
mation or memory increase over the path [20], [21]. However, such approaches fit well and have been mainly applied in static scenarios. In dynamic environments their performance decays significantly.

Experimental studies of the role of the mammalian hippocampus in spatial navigation inspired the concept of so called cognitive maps [22]. Although neurophysiological basis of this phenomenon in big part remains unclear growing experimental evidence and numerical models show that the activity of place cells is involved in navigation constructed over cognitive maps (see e.g. [23], [24]). Briefly, a cognitive map establishes a process that generates an absolute stationary framework constructed from spatial relationships in the agent's environment. Then the agent can virtually move through the map and reach the target. Thus a cognitive map is an essential part of an IR. According to this concept, cognitive navigation exploits spatial relations among objects in the map to extract information necessary to move in the environment.

Many of the existing cognitive approaches to navigation are based on topological maps. They represent the environment as a graph with vertices and edges describing places and spatial relations among them. This allows finding different routes between two places [25]. However, the agent can only use visited places or concatenate known routes. No novel routes (e.g. shortcuts) can be generated in unexplored terrains. Besides, different places must be clearly distinguished to be mapped into different nodes and at the moment no satisfactory solution exists to tackle this problem [25], [26]. Some of these limitations have been overcome by survey navigation, which embeds the perceived elements and their spatial relations into a common system of reference. Then the robot can deduce new spatial correlations and find novel trajectories. Advantages of both topological and survey navigations have been exploited in combined approaches [27].

Potentially, cognitive maps can provide sophisticated solutions. However, the majority of the approaches are essentially local and their advantages have been mostly reported in static scenarios. In changing environments Yan and colleagues [28] have shown that cognitive maps permit local in time and space adaptation of the robot's path. In general, local approach to the path planning in dynamic environments is a common trend (see [29] for review). The proposed strategies range from evolutionary APFs [30] to self-organizing and recurrent neural networks [31], [32], passing through biologically-inspired models for planning obstacle avoidance [33].

Local restrictions (see e.g. [34] for discussion) are usually justified by arguing that the dynamics of objects in the environment is often unknown. Hence the agent can rely on movements defined locally in space and time only. Nevertheless in the majority of realistic situations (but see [15]) trajectories followed by at least inanimate objects can be predicted quite accurately. Then anticipation is a vital ability of animals, genetically coded and refined by experience and training [35]. Thus cognitive navigation in animals challenges the local approach. In order to model the behavior of higher living beings we must call upon complementary cognitive models capable of global internal representation of dynamic situations.
Global solutions require prediction of the spatiotemporal dynamics of the objects that can interact with the agent. Different aproaches including extended particle swarm optimization [36], genetic algorithms [37], ant colony optimization [38] have been proposed. However, when searching trajectories alternative to the optimal path, these approaches exhibit relatively poor performance and low versatility (see [39] and references therein). The majority of these algorithms assume immobile target and impose restrictions on the shape of obstacles.

Finally a subtle but critical point for real autonomous behavior concerns another aspect of global solutions. According to Conn and Kam [40], a global approach finds trajectories to the target when they exist but also must detect situations when no solution can be found. Thus the ability of detecting deadlock situations is a critical difference between local and genuine global approaches and the key for significant benefits offered by global strategies in Nature. Indeed, an agent, e.g. a predator, incapable of identifying a priori impractical solutions will waste energy and resources putting its survival at risk.

Recently, for an efficient global description of complex dynamic situations we introduced the concept of Compact Internal Representation (CIR) [41]. CIR can be considered as a global, in the sense of Conn and Kam, cognitive map constructed over specific APF. The idea behind CIR stems from the state-of-the-art cognitive maps and definition of cognition as a construction of spatial relations among static objects. CIR generalizes spatial relations to a new type of effectively static elements existing in a special representation of dynamic environments. Potential collisions of the agent, obstacles, and targets are modeled by a dynamical system and then the detected critical events are mapped into static IR. Thus CIR compacts essentially time-evolving situations and represents them as a static map containing information necessary for navigation. Therefore it solves both above-stated questions of the IR of dynamic situations.

Earlier we showed that CIRs can be easily stored and then retrieved from an associative memory [42]. This provided a theoretical basis for building an agent mimicking cognitive abilities of higher animals. Such agent must possess "conscious" and "subconscious" pathways. While the conscious pathway generates CIRs for novel situations, the subconscious one learns experiences (situations and corresponding CIRs) for further use in a quick and efficient manner. Thus the agent acquires the ability of responding "without thinking" to familiar situations.

In this work we further develop our concept, extend it to mobile targets, and provide a novel neural network architecture that now unifies understanding of the environment (conscious pathway) and learning of experiences (subconscious pathway) in a closed autonomous loop. By default the agent relies on the subconscious pathway. If a perceived situation is identical to one of the previously experienced or similar but not identical (due to e.g. sensory uncertainty) then the subconscious pathway is able to provide an effective solution. However, if it fails to solve the problem, then the conscious pathway is activated and generates solution to this significantly novel scenario. To prove the feasibility of the CIR theory and 
of the proposed architecture we build a setup and perform experiments with roving robots. Our data show that indeed the CIR-based cognitive neural architecture provides the agent with reliable and flexible behaviors. We also demonstrate the robustness of the approach to random fluctuations in the sensory information, which is a must for outdoor applications.

\section{EXPERIMENTAL SETUP}

In order to study the problem of cognitive near-range navigation we built a setup (Figs. 1 A and 1 B) with roving robots simulating an agent, one target, and two moving obstacles in a white arena $(150 \times 150 \mathrm{~cm})$. The programmable robots (Moway, Minirobots S.L., Fig. 1A) were controlled through a WiFi interface with customary written $\mathrm{C \#}$ code managed by Matlab (R2011a 64-bit, The MathWorks, Inc.) running on a standard PC. Each robot can freely move with the velocity ranging between 35 and $75 \mathrm{~cm} / \mathrm{s}$. The robot performing as a cognitive agent moved with constant velocity $56 \mathrm{~cm} / \mathrm{s}$, while obstacles had the velocities $45 \mathrm{~cm} / \mathrm{s}$ and $70 \mathrm{~cm} / \mathrm{s}$. In order to distinguish objects in the arena, black cardboard figures were stuck over each robot (Fig. 11B): arrow-shaped over the agent, circular shapes over the obstacles, and a strip over the target. The visual information in the arena were captured by a zenithal camera (Logitech, QuickCam Communicate STX). For object recognition we used standard routines from the Matlab Image Processing Toolbox. The snapshots of the arena were taken at $50 \mathrm{~Hz}$ rate. Then all black objects were identified in each frame. The objects were differentiated (agent, obstacles, and targets) by the size of the black figures stuck over the robots. The displacement of centroids were used for object tracking and for determining their positions, velocities, and accelerations using finite difference approximation.
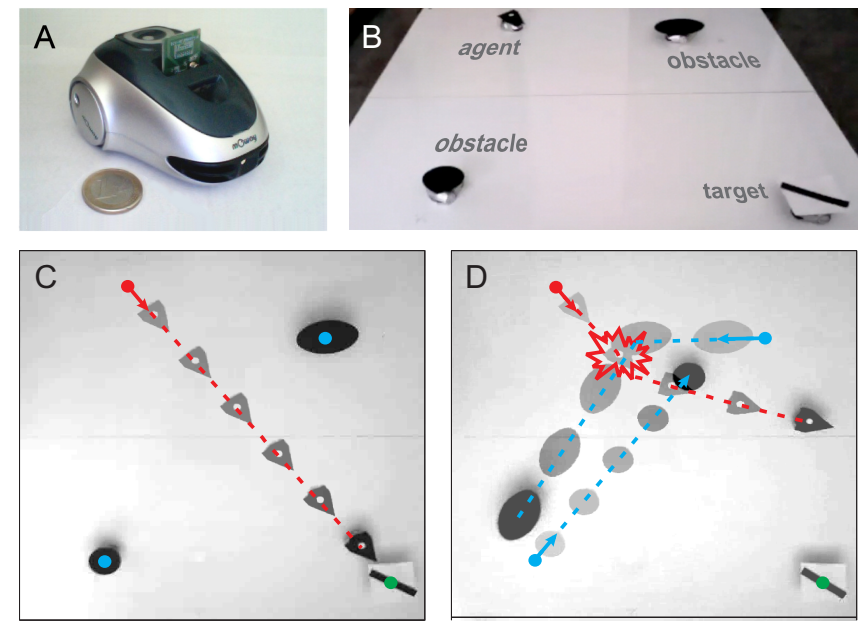

Fig. 1. Experimental setup. A) Roving robot equipped with WiFi interface aside of a $1 €$ coin. B) Side view of a $(150 \times 150)$ $\mathrm{cm}$ arena with four robots simulating an agent (arrow shape), two obstacles (circular shapes), and a target (stripe). C) Top view of a static situation (captured by a zenithal camera). The agent has a goal to reach the target. Six consecutive frames marking the agent's positions are shown superimposed. D) Dynamic situation. A collision occurs if the agent makes the decision from the initially perceived visual information as in the static case (dots and arrows mark initial positions and velocities, respectively).
Figures $1 \mathrm{C}$ and 1D show examples of static and dynamic situations, respectively. In the static environment (Fig. 1 $1 \mathrm{C}$ ) the visual information obtained at the initial time instant determines the motor decision. The agent moves along a straight trajectory and easily reaches the target. In a similar but dynamic situation both obstacles cross the agent's path (Fig. 1D). Then the same agent's behavior would lead to a collision. Thus this situation requires more sophisticated path planning based on anticipation of the state of the environment in the future. In the following sections we shall show how the CIR concept can be used for this purpose.

\section{TRAJECTORY MODELING NEURAL NETWORK}
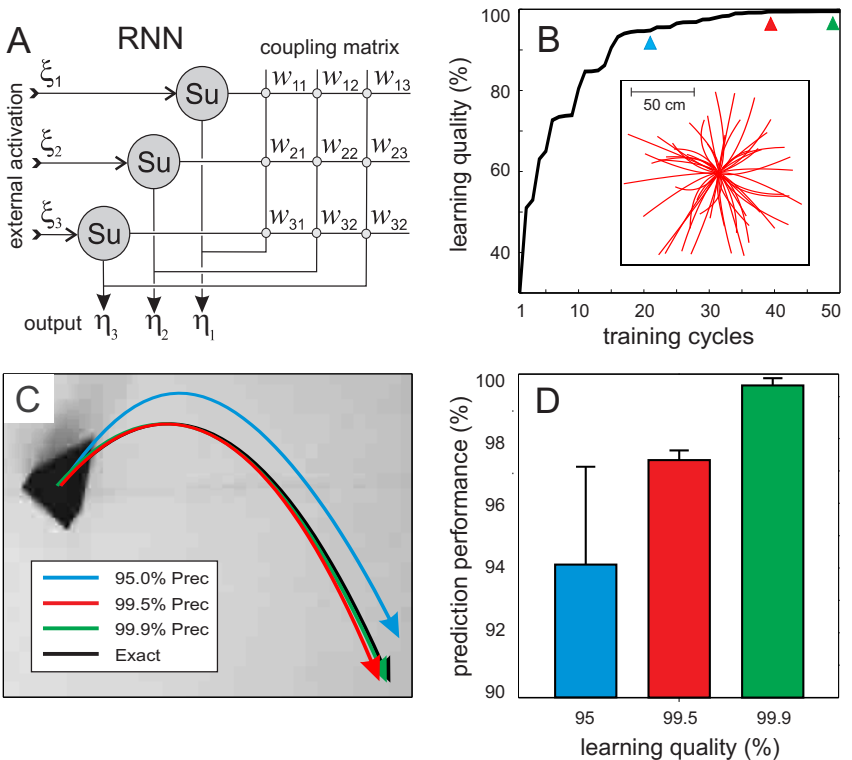

Fig. 2. Prediction of trajectories of moving obstacles and targets. A) Sketch of a recurrent neural network used for trajectory modeling. B) Learning performance. Blue, red, and green triangles mark the training quality with $d=95 \%, 99.5 \%$, and $99.9 \%$. Inset shows 50 (parabolic) trajectories used for training the TMNN. C) Prediction of an experimental trajectory at different levels of the learning quality, corresponding to triangles in (B). D) Mean and standard deviation of the performance of the TMNN in predicting trajectories of 100 objects for three different values of the learning quality.

Generation of a CIR requires prediction (in the agent's mental time $\tau$ ) of the trajectories of near-range objects in the arena. This task is performed by the Trajectory Modeling Neural Network (TMNN). Figure 2 A shows the implementation of the TMNN. It consists of three recurrently coupled neurons with external input $\xi(k) \in \mathbb{R}^{3}$ and output $\eta(k+1) \in \mathbb{R}^{3}$, where $k$ denotes discrete mental time (i.e. $\tau=k h$, where $h$ is the time step) [43]. The dynamics of the network is given by

$$
\eta(k+1)= \begin{cases}\xi(k), & \text { if }|\xi(k)|>\delta \\ W \eta(k), & \text { otherwise }\end{cases}
$$

where $W \in \mathcal{M}_{3 \times 3}(\mathbb{R})$ is the coupling matrix and $\delta>0$ is the tolerance constant, which we introduced in this work to avoid the noise instability ( $\delta=10^{-6}$ in experiments). To 
model trajectories in 2D we need two identical networks (or one with six units) for $x$ and $y$ components.

For the sake of simplicity we assume that trajectories of all objects in the environment can be described by quadratic functions of time (prediction of more complex trajectories is also possible, but see [41] for discussion). In order to train the TMNN to recognize such routes we generated a set of 50 random parabolic trajectories (Fig. $2 \mathrm{~B}$, inset) and presented them to the network as external input in the form $\xi(k)=(x(k), v(k), a(k))^{T}$, i.e. the first three dynamic moments of the trajectory (the same for $y$-component). The velocity and acceleration are given by the finite differences: $v(k)=(x(k)-x(k-1)) / h$ and $a(k)=(v(k)-v(k-1)) / h$, respectively. Note that such approximation does not introduce additional error due to appropriate design of the network [41]. The interneuronal couplings are updated during the learning according to

$$
\begin{aligned}
W(k+1)= & W(k)\left(I-\varepsilon \xi(k-1) \xi^{T}(k-1)\right) \\
& +\varepsilon \xi(k) \xi^{T}(k-1)
\end{aligned}
$$

where $\varepsilon>0$ is the learning rate.

Under proper learning rate $\varepsilon<\varepsilon^{*}, W$ converges to a theoretical matrix $W_{\infty}$ (see for details [43]). The distance

$$
d(k)=100 \%\left(1-\frac{\left\|W(k)-W_{\infty}\right\|}{\left\|W_{\infty}\right\|}\right)
$$

is used to quantify the learning performance (Fig. $2 \mathrm{~B}$ ). Indeed, under training the interneuronal couplings quickly converged (in less than 50 cycles) to the theoretically predicted values. For further analysis we selected the coupling matrices $W_{95}$, $W_{99.5}$, and $W_{99.9}$ corresponding to different learning quality $d=95 \%, 99.5 \%$, and $99.9 \%$, respectively.

Once the training process is deemed finished, the TMNN can be used to predict novel trajectories. The camera captures the first three instants (frames) of the object's movement and the obtained information is introduced in the TMNN as an external input consisting of the initial position, velocity, and acceleration of the object $\xi(0)=\left(x_{0}, v_{0}, a_{0}\right), \xi(k)=0$ for $k=1,2, \ldots$ Then the TMNN generates the following object's trajectory by iterating Eq. (1). Figure $2 \mathrm{C}$ shows top-view of a robot in the arena following the black curve and the robot's trajectories predicted by the TMNN for the three different values of the learning quality.

In order to quantify the TMNN prediction performance we used the Fréchet distance $d_{F}(\gamma, \tilde{\gamma})[44]$ measuring the similarity between the original robot trajectory $\gamma$ and trajectory predicted by the TMNN $\tilde{\gamma}$. Then the prediction performance is

$$
P(\gamma, \tilde{\gamma})=100 \%\left(1-\frac{d_{F}(\gamma, \tilde{\gamma})}{l(\gamma)}\right)
$$

where $l(\gamma)$ denotes the length of the curve $\gamma$. Figure 2D shows the statistics of the prediction performance for different values of the learning quality obtained for a set of random parabolic trajectories $\left\{\gamma_{i}\right\}_{i=1}^{100}$. The learning quality achieved in about 50 training cycles is enough to obtain practically $100 \%$ fidelity in the prediction of trajectories by the TMNN.

\section{CONCEPT OF COMPACT INTERNAL REPRESENTATION}

Let us briefly recall how a CIR of a dynamic situation can be created and then used for navigation [41], [42]. CIR is generated by a reaction-diffusion process taken place in Causal Neural Network $(\mathrm{CNN})$, a $60 \times 60$ square lattice of modified FitzHugh-Nagumo neurons described by:

$$
\begin{aligned}
& \dot{r}_{i j}=q_{i j}\left(H\left(r_{\mathrm{th}}-r_{i j}\right)\left[f\left(r_{i j}\right)-z_{i j}\right]+d \Delta r_{i j}-r_{i j} p_{i j}\right) \\
& \dot{z}_{i j}=\left(r_{i j}-7 z_{i j}-2\right) / 25
\end{aligned}
$$

where $r_{i j}$ and $z_{i j}$ are the potential and recovering variable of the $i j$-th neuron, respectively. Dots in Eq. (5) represent derivatives in respect to the mental time $\tau, \Delta$ is the discrete Laplacian, $d$ is the diffusion constant, $H$ is the Heaviside function, $r_{\text {th }}=3$ is the phase threshold, and $f(r)$ is a cubiclike FitzHugh-Nagumo nonlinearity, which we set to $f(r)=$ $\left(-r^{3}+4 r^{2}-2 r-2\right) / 7$ in all experiments. The size of the lattice scales to the size of the arena (i.e. the interneuron distance is assumed to be equal to $2.5 \mathrm{~cm})$. Functions $q_{i j}(\tau)$ and $p_{i j}(\tau)$ (equal to one and zero, respectively, at the beginning of the simulation) describe the movement of obstacles and targets, respectively, and will be described in detail below. The time evolution of the variables $\left\{r_{i j}\right\}_{i, j=1}^{60}$ creates a 2-D potential field that forms CIR. Further on we shall denote the resulting CIR by

$$
c=\left\{r_{i j}\right\}_{i, j=1}^{60} \in \mathcal{M}_{60 \times 60}(\mathbb{R})
$$

Let us now consider a dynamic situation similar to that shown in Fig. 1D. The agent (Fig. 3A, blue circle) should move with a constant velocity and reach the mobile target (red area) avoiding the moving obstacles (black areas). The initial conditions, i.e. objects' positions, velocities, and accelerations, are supplied to the TMNN that simulates the obstacles and target's trajectories (see Sect. III).

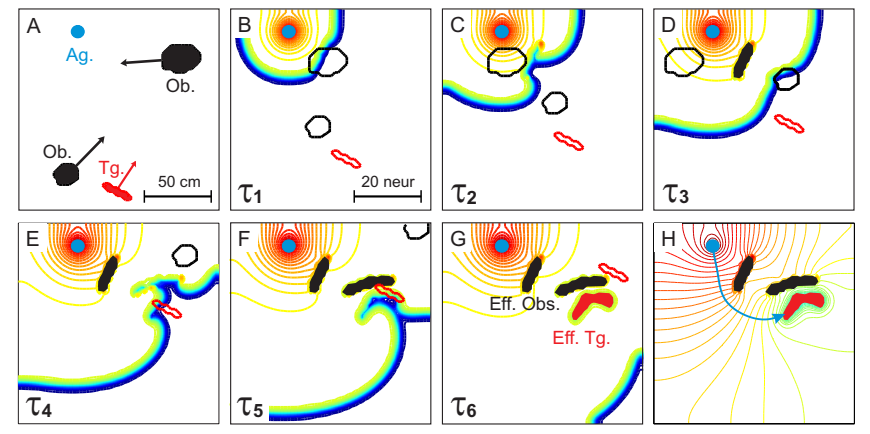

Fig. 3. Generation of Compact Internal Representation of a dynamic situation. A) Initial configuration of the arena. The agent (Ag), target $(\mathrm{Tg})$, and obstacles $(\mathrm{Ob})$ are shown as blue circle, red elongated area, and black oval-like areas, respectively. Arrows mark their velocities. B)-G) Sequential snapshots of the CNN state (the pattern $\left\{r_{i j}(\tau)\right\}_{i, j=1}^{60}$ is plotted). Interaction of the wave front (expanding blue curve) with the objects generates an effective target and obstacles (red [Eff Tg] and black [Eff Obs] filled areas, respectively). Virtual positions of the target and obstacles are shown by contour shapes. $\mathrm{H})$ The potential field $c$ represented by isolines provides CIR of the considered dynamical situation. Blue arrowed curve shows the shortest trajectory to the effective target and thus ensures interception of the target moving in the arena avoiding collisions with obstacles. 
Simultaneously with the TMNN in the CNN a circular wavefront is initiated at the agent's location (Fig. 33B). Propagation of the wavefront in the lattice mentally simulates all possible positions of the agent at each moment in the virtual future. Figures $\sqrt{3} \mathrm{~B}-3 \mathrm{G}$ show sequential snapshots of the $\mathrm{CNN}$ state. For $\tau=\tau_{1}$ the first contact of the wavefront and one of the (moving) obstacles occurs (Fig. 3B). This contact marks the place where the agent would collide with the obstacle if the corresponding trajectory were performed in the arena. The cells of the CNN corresponding to those locations are frozen, i.e. in the network (5) $q_{i, j}(\tau)=0$ for $\tau \geq \tau_{1}$, $(i, j) \in \Omega_{B}$, where $\Omega_{B}$ is the set of wavefront-obstacles collisions. $\Omega_{B}$ constitutes an effective obstacle, i.e. a static structure containing the critical spatiotemporal information concerning potential collisions between the agent and the obstacle (Fig. 3D, area filled in black). We note that in general an effective obstacle has a shape different from the shape of the corresponding real obstacle. Therefore the problem of avoidance of moving obstacles in the arena is reduced to the avoidance of static effective obstacles created in the mental map.

In the same fashion interaction between the wavefront and the (mobile and immobile) targets will produce effective targets. Figures $3 \mathrm{E}-3 \mathrm{G}$ show how cells, where the wavefront and the virtual target collide, are frozen by setting $p_{i, j}(\tau)=1$ for $\tau \geq \tau_{4},(i, j) \in \Omega_{T}$ in the network (5), where $\Omega_{T}$ is the set of wavefront-targets collisions. Thus $\Omega_{T}$ forms an effective target (Fig. $3 \mathrm{G}$, area filled in red). We note that in general one target can create several effective targets (disjoin set $\Omega_{T}$ ), which enables different strategies for target catching.

In the region behind the wavefront passive diffusion (controlled by the Heaviside term in (5) ) creates a static potential field $c$, which includes the agent position and the effective obstacles and targets. This potential field, i.e. a 2D pattern $\left\{r_{i j}\right\}$, is the CIR for the considered dynamic situation (Fig. $3 \mathrm{H})$. This field can be used to draw possible trajectories from the initial agent's position to the effective targets by a gradient descend method (in the simplest form: $\gamma_{j+1}=\gamma_{j}-\nabla r$ ). Following such trajectories in the arena ensures avoiding obstacles and catching the target.

Finally we note that the performance of CIR generation does not depend on the complexity of the environment. It takes about the same time to generate CIR of an empty arena and CIR of a complex dynamic situation with numerous static and moving objects (for more example see [41], [45]).

\section{Network ARChitecture FOR COGNitive NAVIGATION BASED ON CIR}

Above we discussed how the CIR collapses the time dimension of a dynamic situation by mapping only critical events (virtual collisions and effective targets) into a static cognitive map. Therefore CIR of a dynamic situation is a static pattern (i.e. an $(n \times n)$-matrix (6), $n=60$ in our experiments), which can be learned, stored in memory, retrieved, compared, etc. Thus we can easily manage different realistic experiences in a fast and reliable manner. Earlier we discussed how such cognitive abilities can be implemented in a neural network
[42]. Here we propose a closed-loop self-consistent neural network architecture, which enables autonomous living.

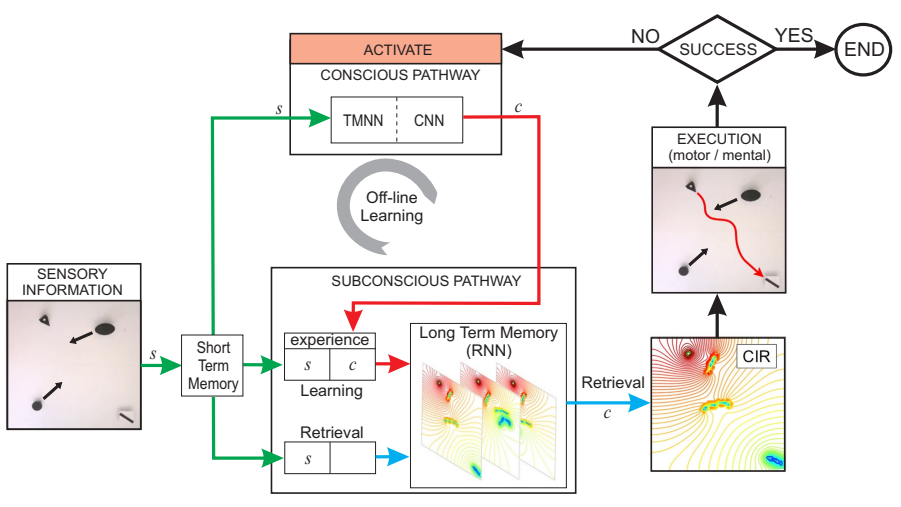

Fig. 4. Block-scheme architecture of a CIR-based cognitive robot.

Figure 4 shows the agent's architecture. Cognition begins with perception of a situation in which the agent is involved. For this purpose in our experimental setup we use visual information captured from a zenithal perspective of the arena. The perceived situation after preprocessing (see Sect. III) is coded in a vector $s \in \mathbb{R}^{6 m}$ consisting of positions, velocities, and accelerations of all objects ( $m$ is the number of objects; $s$ may also include information on the shape of objects). The sensory information $s$ is then stored in a short term memory that replicates it to the "conscious" and "subconscious" pathways.

The output of both pathways is a CIR, $c$. In general the conscious pathway is reliable but slow, whereas the subconscious one produces $c$ much faster but its fitness depends on the previous learning. By default the agent relies on the subconscious pathway. The CIR $c$ retrieved from the Long Term Memory (LTM) is used for navigation (Fig. 4). The standard gradient descent method (see Sect. IV) provides a trajectory that, transformed in motor orders, permits the agent to reach the target (Fig. 4, red curve in the arena). We note that the execution may be also mental, i.e. no action is taken in real world.

Next action is determined by the standard "trial and error" approach in learning (see e.g. [46]). If the motor execution was successful then we assume that the task was accomplished and no further action is required. If the agent fails to safely reach the target, then the process of learning is activated. Failure may occur if the observed situation is significantly novel for the agent and it has no adequate CIR stored in the LTM, or due to the sensory uncertainty. As we shall show below the LTM is robust again small uncertainties. Thus if the situation is similar enough to one of the previously experienced scenarios, then the LTM is capable of generating effective CIR and the agent succeeds in catching the target.

The process of learning occurs offline, similarly to the memory consolidation during sleep. The conscious pathway produces reliable CIR, $c$, for a given sensory vector $s$ (see Sects. III and IV]. This CIR is fed back into the subconscious pathway, where a recurrent neural network (implementing LTM) establishes associations between the sensory vector $s$ and the CIR $c$. This dynamical process takes place several times until the experience will be learned. Then if the agent 
faces one of the learned situations (or one similar enough), the subconscious pathway retrieves the corresponding CIR in a fast and reliable way. We note that the LTM can be preprogrammed for stereotypic experiences. Then the agent could successfully deal with stereotypic situations from the beginning.

\section{A. Conscious pathway}

The perception of a significantly novel (not learned) situation forces the agent to understand it by creating "consciously" the corresponding CIR (Fig. 4, conscious pathway). This CIR must be adequate, otherwise the agent will never learn how to cope with the given situation. Thus we tested the quality of CIRs created by the conscious pathway for real robot navigation.
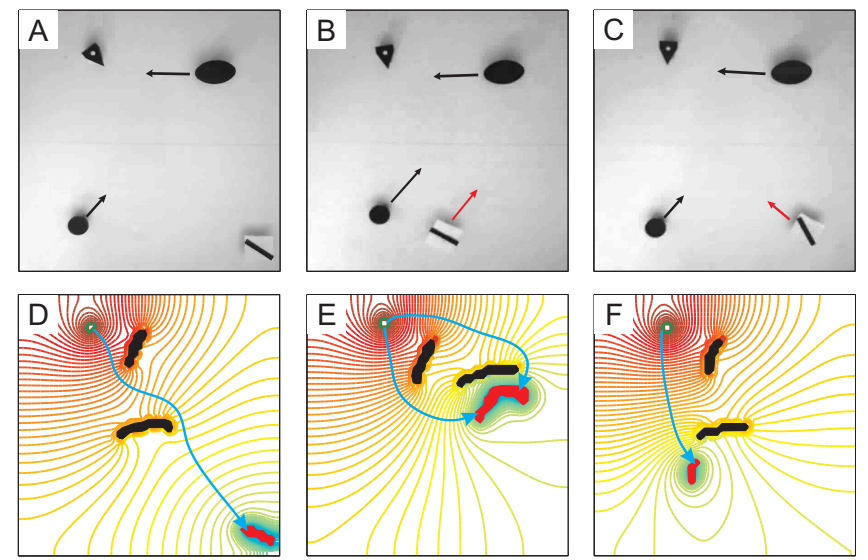

Fig. 5. Experimental navigation based on CIR provided by conscious pathway for three different time-evolving situations $S_{1}, S_{2}$, and $S_{3}$. A)-C) Initial configurations for each dynamic situation. Arrows indicate the initial velocities and directions of the objects in the arena. D)-F) The corresponding CIRs created by the "conscious" pathway. Blue curves show trajectories to the effective target without collisions with the obstacles.

In order to illustrate the capability of CIR to represent dynamic situations three different time-evolving environments were considered (Figs. $5 \mathrm{~A}-5 \mathrm{C}$ ). The target is immobile in situation $S_{1}$, whereas in situations $S_{2}$ and $S_{3}$ it moves in different directions. In all three situations two obstacles cross the agent's path to the target. Zenithal camera captured $0.5 \mathrm{~s}$ initial interval of the evolution of the robots representing the obstacles and the target. The initial conditions (sensory vector $s$ ) have been used to mentally simulate the target's and obstacles' trajectories (Sect. III). Finally this information served for generating CIR as described in Sect. IV and illustrated in Fig. 3 for $S_{2}$.

Figures $5 \mathrm{D}-5 \mathrm{~F}$ show the CIRs for $S_{1}, S_{2}$, and $S_{3}$, respectively. Note the different shapes and locations of effective targets and obstacles reflecting distinct dynamical circumstances. Blue pathways in Figs. 5D and 5F represent the shortest trajectories solving the corresponding navigation problems for situations $S_{1}$ and $S_{3}$, respectively. The CIR in Fig. $5 \mathrm{E}$ $\left(S_{2}\right)$ admits two trajectories of about the same length. The difference between them is the distinct collision risk to reach the moving target. Nevertheless all trajectories in cognitive maps shown in Figs. 5D-5F lead the agent to the target with no collision against obstacles (experimental videos are available at [45]). Thus the conscious pathway indeed provides correct CIRs for dynamic situations.

\section{B. Subconscious pathway}

When the agent faces a familiar situation, the sensory information, $s$, passes through the subconscious pathway, which retrieves the corresponding CIR, $c$, from the associative long term memory (Fig. 4).

The general RNN introduced in Sect. III (Fig. 2A) can also learn static patterns [43], [47], [48]. Since CIRs after all are static matrices linked to specific initial sensory information the same RNN but with higher number of neurons can implement a suitable associative memory.

We shall call an experience the union of the initial sensory information for a dynamic situation $s \in \mathbb{R}^{6 m}$ and the respective CIR $c \in \mathcal{M}_{n \times n}(\mathbb{R})$. We order each CIR into a $1 \mathrm{D}$ vector $c \in \mathbb{R}^{n^{2}}$. Then each experience is a composite vector

$$
u=(c, s)^{T} \in \mathbb{R}^{n^{2}+6 m}
$$

simultaneously describing the situation and the corresponding CIR. Finally the associative memory is a recurrent neural network shown in Fig. 2A with $\left(n^{2}+6 m\right)$ neurons. This network first goes through the learning and then can be used for retrieval of CIRs.

1) Learning phase: Learning phase (offline in general) is implemented through sequential presentations to the RNN of a set of $M$ experiences $\left\{u_{i}\right\}_{i=1}^{M}$. At each learning step $k$ the network is exposed to one of the composite vectors $u_{i}$ and the coupling matrix is updated according to

$$
W(k+1)=W(k)\left(I-\varepsilon \xi(k) \xi^{T}(k)\right)+\varepsilon \xi(k) \xi^{T}(k)
$$

where $\varepsilon$ is the learning rate and $\xi(k) \in\left\{u_{i}\right\}_{i=1}^{M}$ is an element from the set of experiences. Earlier we have shown that there exists $\varepsilon^{*}>0$ such that for $0<\varepsilon<\varepsilon^{*}$ the learning process converges [43]:

$$
W\left(u_{1}, \ldots, u_{M}\right)=\lim _{k \rightarrow \infty} W(k)
$$

The limit coupling matrix (9) is given in Ref. [42].

Theoretically the associative memory can store up to $n^{2}+$ $6 \mathrm{~m}$ experiences. We also note that the order of presentation of experiences is not essential [43]. Thus different experiences can be learned sequentially or in parallel.

2) Retrieval phase: Once the learning phase has been finished the RNN can be used to associate new sensory information $s_{l}$ with a CIR $c_{l}$. The corresponding CIR appears on the output of the RNN.

For convenience we split the coupling matrix 9 in four blocks:

$$
W=\left(\begin{array}{ll}
W_{c} & W_{a} \\
W_{b} & W_{s}
\end{array}\right)
$$

where $W_{c} \in \mathcal{M}_{n^{2} \times n^{2}}, W_{a} \in \mathcal{M}_{n^{2} \times 6 m}, W_{b} \in \mathcal{M}_{6 m \times n^{2}}$, and $W_{s} \in \mathcal{M}_{6 m \times n^{2}}$. We also represent the output of the RNN as $\zeta(k)=\left(\zeta_{c}(k), \zeta_{s}\right)^{T}$, where $\zeta_{c} \in \mathbb{R}^{n^{2}}$ and $\zeta_{s} \in \mathbb{R}^{6 m}$ are parts corresponding to CIR and sensory information, respectively. 
During retrieval we present to the RNN (and maintain at the input) the sensory part of one of the learned experiences. Then the network activation is given by

$$
\xi(k)=(\underbrace{0,0, \ldots, 0}_{n^{2}}, s_{l})^{T} \quad k \geq 0
$$

Consequently the last $6 m$ neurons in the RNN have no dynamics: $\zeta_{s}(k)=s_{l}, k \geq 0$, while the others follow the linear map:

$$
\zeta_{c}(k+1)=W_{c} \zeta_{c}(k)+W_{a} s_{l}
$$

It has been proven that following this scheme the LTM completes the missing part of $u_{l}[42]$ and hence retrieves the CIR

$$
c_{l}=A s_{l}
$$

where $A=\left(I-W_{c}\right)^{-1} W_{a}$. We note, that according to (12) the subconscious pathway provides a CIR for any (even completely novel) sensory information. However, if the agent (matrices $W_{c}$ and $W_{a}$ ) did not learned previously an adequate experience, then the retrieved CIR may be useless.

\section{Vi. Performance of Subconscious pathway}

Success of navigation in dynamic situations depends on the velocity and quality of CIR's retrieval, and hence on the performance of the associative long term memory.

\section{A. Offline learning of experiences}

To simulate the process of learning and retrieval we used the three dynamic situations $S_{1}, S_{2}$, and $S_{3}$ shown in Figs. 5.A$5 \mathrm{C}$ and the corresponding CIRs (Figs. 5D-5F) generated by the conscious pathway. Then we composed three experience vectors $u_{i}=\left(c_{i}, s_{i}\right), i=1,2,3$ and presented them several times to the subconscious pathway for offline learning (Fig. 47. After learning we examined how the agent solves the navigation problem by presenting each one of the three dynamic situations $\xi(k)=\left(0, s_{i}\right)$ and checking the retrieved CIRs, $\hat{c}_{i}$.

Figure 6 illustrates the retrieval results for each dynamic situation (columns) after 2, 6, 70, and 220 training cycles (rows). Two learning cycles is insufficient for navigation, no trajectory to the target can be traced. CIRs retrieved after 6 training cycles reveal mixtures of the conscious CIRs and also cannot be used for tracing correct trajectories to the target. Thus at the beginning of the training the agent tends to confuse different experiences and as a consequence it cannot successfully solve the navigation problem. Keeping training, the quality of CIRs is refined (Fig. 6. $N_{t r}=70$ cycles) and after 220 training cycles the CIRs retrieved from the memory, $\hat{c}_{i}$, are practically identical to the conscious CIRs, $c_{i}$, (compare Fig. 6 , bottom row vs Figs. 5D-5F).

We notice that CIRs $\hat{c}_{1}$ and $\hat{c}_{3}$ were practically indistinguishable for up to 70 training cycles. On the contrary $\hat{c}_{2}$ was clearly different starting from early stages of the learning. This illustrates the main impact of the similarity among experiences: at the beginning the agent tends to fuse (and sometimes confuse) similar experiences and separate different ones. Fine separation of similar situations requires

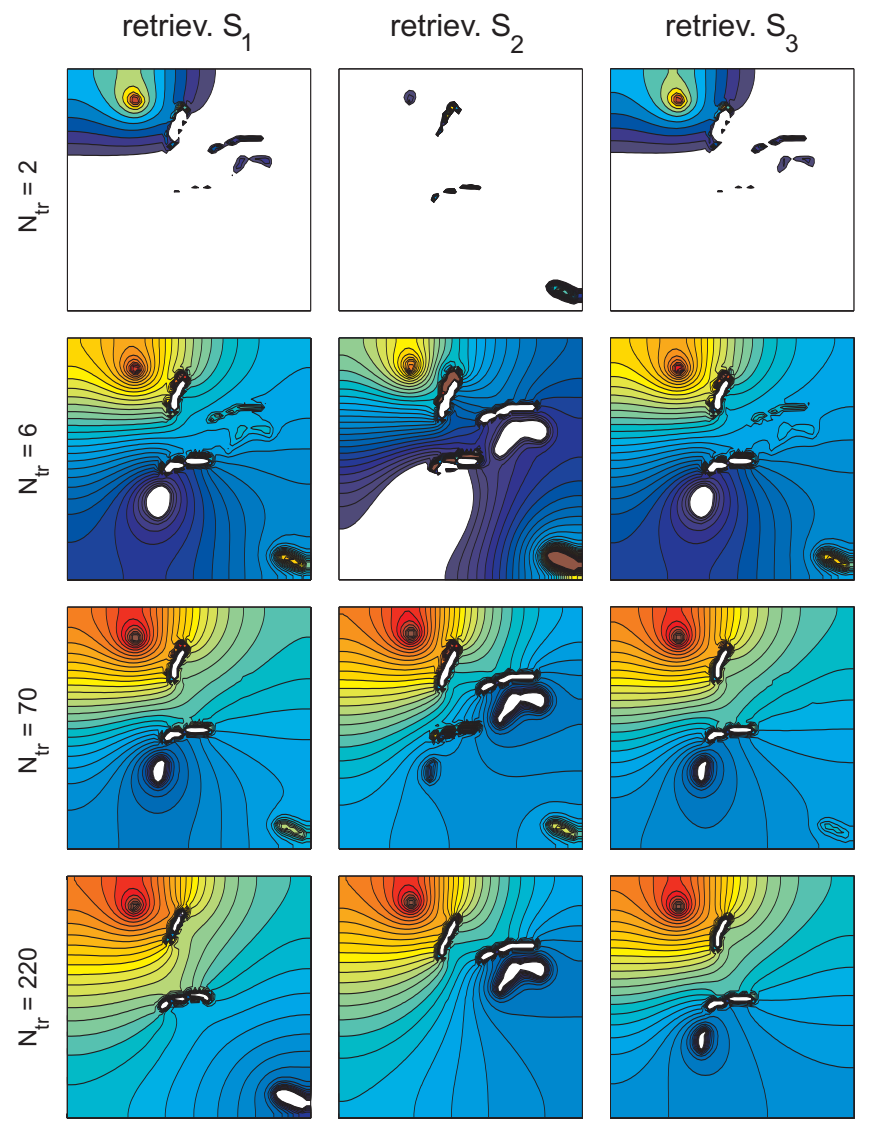

Fig. 6. Learning of experiences and retrieval of CIRs from the subconscious pathway. Each experience, i.e. the sensory information $s_{1,2,3}$ and the CIRs $c_{1,2,3}$ corresponding to the dynamic situations $S_{1,2,3}$ (Fig. 5), has been learned and then retrieved from the memory. Rows correspond to retrieval after $N_{\mathrm{tr}}=2,6,70$ and 220 learning cycles. At the beginning the quality of retrieved CIRs is insufficient for navigation. The quality improves with training cycles and for $N_{\mathrm{tr}}=220$ the CIRs retrieved from the memory are identical to conscious CIRs (Fig. 5).

longer learning. However, it may not always be necessary, i.e. single solution for similar problems may perfectly work.

Thus the learning of different experiences converges and the subconscious pathway can finally provide real benefit. Indeed, the "conscious" processing of a dynamic situation on a standard PC implemented in Matlab R2011a lasts around 27 s, while the subconscious pathway provides the same CIR in less than $0.4 \mathrm{~s}$, which is of order of the human reaction. We note that these numbers can be significantly reduced (by several orders of magnitude) by using parallel multicore calculations (e.g. using CUDA with GPUs) or dedicated hardware implementation. In fact, the $60 \times 60$ lattice used in the conscious pathway for creating CIRs has 3600 neurons. Evaluating their dynamics in parallel enables up to 3600 folds acceleration. For example, our FPGA implementation of the conscious pathway provides CIRs in less than 0.25 s (i.e. about 100 folds faster than on a PC) [49].

\section{B. Dynamics of CIR retrieval}

Let us now illustrate how the learned experiences are retrieved from the associative memory. Each CIR can be 
considered as a point in a multidimensional space $\left(c \in \mathbb{R}^{n^{2}}\right)$. Then the curse of dimensionality is the major obstacle for establishing quantitative relations among different experiences. For dimension reduction we used the standard Principal Component Analysis (PCA) [50] applied to CIRs.

The first two principal components (PCs) captures the most significant information in the set of three CIRs. Figure 7 illustrates the evolution of three CIRs $c_{1,2,3}(k)$ in the PC-space during retrieval (see also Sect. V-B2). The retrieval always starts from a blank CIR (Fig. 7, black point in the origin). Then in one iteration CIRs jump into different corners of the $\mathrm{PC}$-space. The CIR $c_{2}(k)$ corresponding to $S_{2}$ always stays far a way from the other two along $\mathrm{PC}_{1}$ and converges rapidly to $c_{2}$. CIRs corresponding to the similar situations $S_{1}$ and $S_{3}$ approach each other at the beginning (dark blue points). Thus at intermediate stage of the retrieval process (iteration $k=15$ ) the associative memory tends to return practically the same CIRs for $S_{1}$ and $S_{3}$. A longer retrieval is required to separate these experiences and to distinguish $c_{1}(k)$ and $c_{3}(k)$ properly (red points, $k \in[50,60]$ ). This insight together with the results of the previous subsection show that both learning and retrieval of CIRs for similar situations demand longer processing.

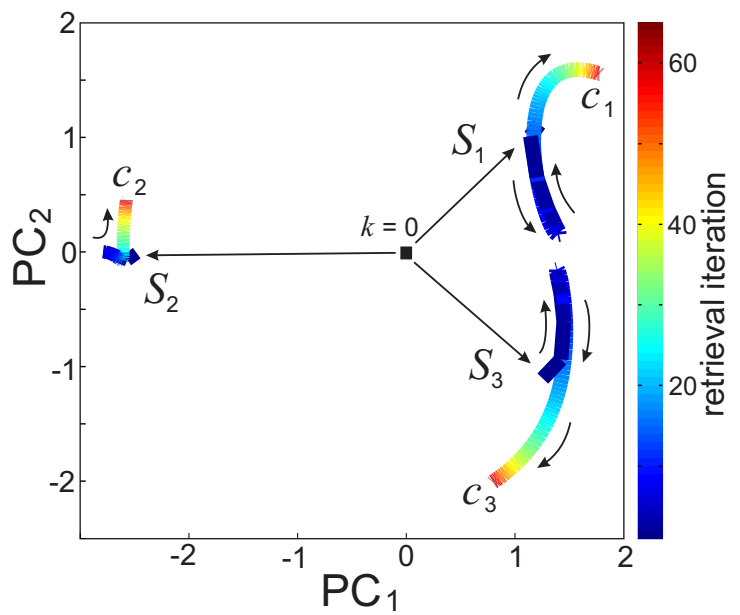

Fig. 7. Dynamics of the CIR retrieval on the plane of first two principal components (PCs). CIRs corresponding to experimental situations $S_{1,2,3}$ (Figs. 5A-5 C) are retrieved from the associative memory. Each retrieval iteration, $k$, is color coded. The retrieval process starts at the blank CIR $(k=0)$ and converges (in $k \approx 60$ iterations) to $c_{1,2,3}$ (see also Figs. $5 \mathrm{P}-5 \mathrm{~F}$ ).

\section{Robustness of associative long term memory}

One of the major challenges in outdoor applications is the inherent noise and uncertainty in the sensory information. Then previously learned situation may not match exactly with the perceived one, which may lead to drastic reduction of the agent's performance. Our architecture automatically accounts for this problem and provides robust retrieval of CIRs.

We model the sensory uncertainty by adding white noise to the $i$-th sensory vector $(i=1,2,3)$ :

$$
\tilde{s}_{i}=s_{i}+D \chi
$$

where $\chi=\left(\chi_{1}, \ldots, \chi_{6 m}\right)^{T}$ is a vector of random Gaussian variables $\chi_{j} \sim \mathcal{N}(0,1)$ and $D$ is the noise intensity.
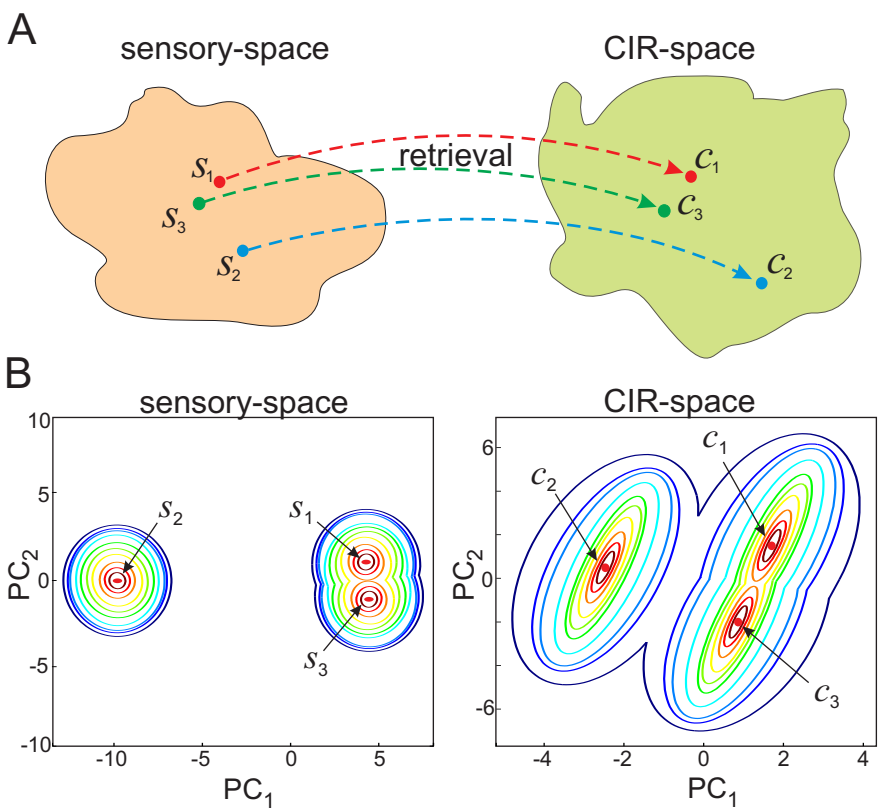

Fig. 8. Robustness of the CIR retrieval under sensory noise. A) Retrieval process as a mapping from the sensory-space into CIRspace. B) Representative example of CIR-retrieval under sensory noise. To reduce dimensions we applied PCA separately over sensory and CIR vectors. Left. Sensory-space. Contour curves correspond to confidence regions (most probable areas for sensory stimuli) for different values of the noise intensity. Colors from red to blue code the noise levels from low to high. For zero noise we have three points corresponding to the noise-free sensory vectors $s_{1,2,3}$ (Figs. $5 \mathrm{~A}-5 \mathrm{C}$ ). Right. The same as left panel but in the CIR-space. CIRs are retrieved by supplying noisy stimuli to the associative memory. $c_{1,2,3}$ are CIRs corresponding to the noise-free sensory vectors $s_{1,2,3}$ (Figs. 5.D-5F).

When a noisy sensory vector $\tilde{s}_{i}$ is perceived, the subconscious pathway provides a CIR $\tilde{c}_{i}$ (Fig. 4), which does not match exactly the learned noise-free CIR, $c_{i}$. The associative long term memory during the retrieval phase performs mapping of sensory stimuli into CIRs:

$$
\begin{gathered}
F: \mathbb{R}^{6 m} \rightarrow \mathbb{R}^{n^{2}} \\
\tilde{s} \mapsto \tilde{c}
\end{gathered}
$$

where the application $F$ is given by 12 . In other words, the RNN takes a vector from the sensory-space $\mathbb{R}^{6 m}$ and maps it into the CIR-space $\mathbb{R}^{n^{2}}$ (Fig. $8 \mathrm{~A}$ ).

We note that the map $F$ is Lipschitz continuous:

$$
\left\|\hat{c}_{1}-\hat{c}_{2}\right\| \leq\|A\|\left\|\hat{s}_{1}-\hat{s}_{2}\right\|
$$

and hence it is uniformly continuous. Thus it is guaranteed that $\hat{c}_{1,2}$ can be as close to each other as we want by requiring only that $\hat{s}_{1,2}$ are sufficiently close to each other. We also note that the CIRs retrieved under noise have no bias:

$$
\mathrm{E}\left[\tilde{c}_{i}\right]=c_{i}, \quad i=1,2,3
$$

where $c_{i}$ are CIRs corresponding to the noise-free sensory vectors $s_{i}$ and $\mathrm{E}[\cdot]$ is the expectation operator. The covariance in the CIR-space is given by:

$$
V=\mathrm{E}\left[\left(\tilde{c}-c_{i}\right)\left(\tilde{c}-c_{i}\right)^{T}\right]=D^{2} A A^{T}
$$


Thus the standard deviation grows linearly with the intensity of the sensory noise. This ensures robust retrieval of CIRs in realistic conditions, i.e. situations similar enough to the learned one will evoke CIRs similar to the learned one.

The eigenvalues and eigenvectors of the matrix $A A^{T}$ define the clustering process of different experiences in the CIRspace. To illustrate this we simulated sensory stimuli corresponding to the situations $S_{1,2,3}$ perturbed by the noise of different intensity $\sqrt{13}$. Figure $8 \mathrm{~B}$ shows the sensory information and retrieved CIRs on the planes of two principal components.

Since the sensory noise is uncorrelated and normally distributed, in the PC representation of the sensory space (Fig. $8 \mathrm{~B}$, left) the confidence regions are superposition of covariance circles. We notice that stimuli corresponding to $s_{2}$ are well separated from those corresponding to $s_{1}$ and $s_{3}$ along PC1 (see also Fig. 77. Besides, the distances $\left\|s_{2}-s_{1}\right\|$ and $\left\|s_{2}-s_{3}\right\|$ are the same. For strong enough noise (yellow to blue curves) the sensory information corresponding to $s_{2}$ and $s_{3}$ (similar situations) can be confused (contour curves overlap). In this case we cannot distinguish either we observe $S_{2}$ or $S_{3}$, no matter the level of learning we reached.

Similar situation is observed in the CIR-space (Fig. 8B B, right). At low enough sensory noise levels (red curves) CIRs retrieved from the memory can be reliably related to the CIRs $c_{1}-c_{3}$, corresponding to the noise-free sensory stimuli $s_{1}-s_{3}$. Thus if the sensory system is capable of distinguishing sensory stimuli then the memory provides correct CIRs. If the noise level growth (yellow to green curves) the sensory system starts confusing the situations $S_{1}$ and $S_{3}$ and consequently the memory cannot provide correct CIRs. However, under extremely strong noise (black curve) the stimulus $s_{2}$ differs from $s_{1}, s_{3}$ (Fig. $8 \mathrm{~B}$, left), while CIRs are mixed up (Fig. $8 \mathrm{~B}$, right). Nevertheless, this is expected given the complex processing of the sensory information, which leads to certain decrease in the signal to noise ratio.

There is another important difference between the sensory and CIR spaces. We note that the pairwise distances $\left\|c_{2}-c_{3}\right\|$ and $\left\|c_{2}-c_{1}\right\|$ are different, i.e. the CIR $c_{2}$ is closer to $c_{3}$ than to $c_{1}$, although in the sensory space the corresponding stimuli are equidistant (Fig. $8 \mathrm{~B}$, left vs right). Mathematically this is due to nonorthogonal mapping of sensory stimuli to the CIR space. From the behavioral view-point it is explained by the fact that paths to the target for situations $S_{2}$ and $S_{3}$ can be quite similar and differ significantly from the path in $S_{1}$ (see Figs. 5D-F). Thus our internal representation is not a simple copy of the external world.

\section{Experimental verification of cognitive navigation}

Let us now present experimental results for the situations shown in Figs. 5- $-5 \mathrm{C}$. Once the sensory information of a specific situation has been processed and the corresponding CIR and trajectories have been obtained, all robots simulating the agent, target, and obstacles (Fig. 1B) are simultaneously activated. For each situation zenithal camera captures the trajectory executed by the agent to compare it with the pathway obtained from the CIR (Figs. 5D-5F).
1) Conscious pathway: Figures $9 \mathrm{~A}-9 \mathrm{C}$ show navigation in the dynamic situations $S_{1}, S_{2}$, and $S_{3}$, respectively. In all situations the agent successfully caught the target and avoided obstacles (see [45] for videos with robot experiments and additional simulations). The reliability of this experimental procedure is quantified by comparing theoretical and experimental trajectories using the measure (4). We obtained the mean deviation of trajectories $2.6 \%$ with the standard deviation $0.18 \%(n=4)$.

Figures 9p shows statistical data for twelve experiments. We quantified the minimal experimental distance from the agent to the obstacles related to the agent's size. This measure describes the safeness of the agent's movements. In average the agent passes no closer than 1.5 agent's size to the obstacles, which is sufficient for most applications. The achievement of the goal is quantified by the minimal distance between the agent and the target. Note that the theoretical distance in this case is equal to zero by construction. The most important source of experimental variability in this measure is the error in robots' initial positions, orientations, and velocities in each navigation experiment (see also Fig. 8). Nevertheless we observe high level of success of cognitive navigation. The average agent proximity to the target is below $30 \%$ of the robot's size, which is again acceptable for most of applications.
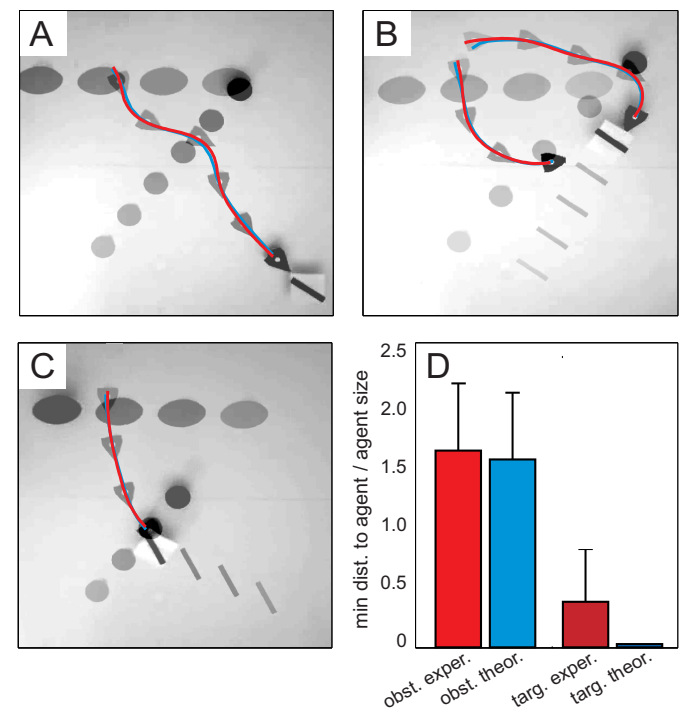

Fig. 9. Robot navigation in dynamic situations. A)-C) Superimposed sequence of snapshots (later frames are darker) for situations shown in Fig. 5 Blue curves show theoretical trajectories and red curves mark the robot pathways. D) Statistical measures of the navigation performance. Two left bars represent means and standard deviations for the minimal distance between the agent and the obstacles (trajectory safeness). Two right bars correspond to the final distance to the target (goal achievement). The distance is given in units relative to the agent's size.

2) Subconscious pathway: The subconscious processing requires a proper learning of the experiences. Figure $10 \mathrm{~A}$ shows the CIR for the situation $S_{1}$ retrieved from the memory after 6 training cycles. Above we showed (Fig. 6) that the CIR obtained at earlier stages of the learning provides fake effective obstacles and targets. Indeed two trajectories obtained from this CIR (Fig. 10A, blue lines) lead the agent to such a 
fictitious target, i.e. the agent fails to catch the target. Figure $10 \mathrm{~B}$ shows experimental trajectories performed by the robot in these conditions. In both cases the robot avoids the obstacles, however, it does not reach the target staying in the right bottom corner. In accordance with our numerical results, at advanced learning stages (i.e. after 200 training cycles) the robot retrieves from the memory high quality CIR and hence follows correct trajectory leading to the target (Fig. 10 C).
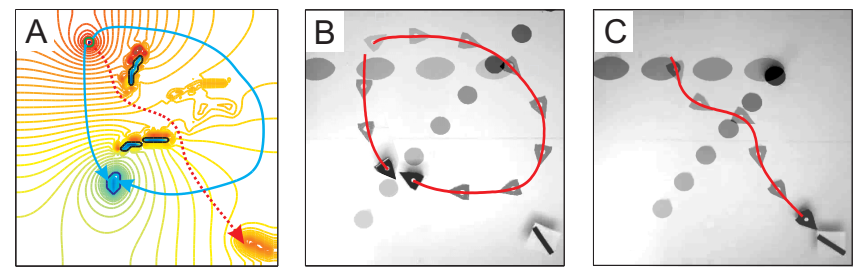

Fig. 10. Robot navigation using CIRs provided by the subconscious pathway at different learning stages. A) CIR corresponding to the situation $S_{1}$ (Fig. 5) retrieved from the long term memory after 6 training cycles. The obtained trajectories (blue curves) lead to a fake target. Red dashed curve shows correct trajectory corresponding to a CIR retrieved after 200 learning cycles. B) Two trajectories (red curves) performed by the robot to the fake target (6 learning cycles), i.e. the robot failed to catch the target. C) The robot successfully catches the target (200 learning cycles).

\section{DISCUSSION}

Navigation in time-evolving environments requires anticipation of possible changes in the external world and appropriate adaptation of the agent's behavior. In general all agent's strategies can be separated in two classes: local and global. Local strategies can be extremely useful when dealing with events unpredictable in time and immediate in space. In turn, global strategies offer significant benefits in predictable environments and also can detect situation when no solution exists. This is why, for example, an efficient capturing of a prey by a predator requires global strategy combined with local rules.

In this work we studied the feasibility of cognitive navigation in realistic environments, based on our recently introduced theoretical framework called Compact Internal Representation. CIR is a specific cognitive map, which provides global solutions to dynamic situations. We have extended the concept on situations with mobile targets. Then using CIR as a core we have proposed a closed-loop neural network architecture consisting of "conscious" and "subconscious" pathways for efficient decision-making. Employing experiments with roving robots and numerical simulations we have shown that the proposed architecture provides the agent with cognitive abilities and allows reliable and flexible navigation in realistic timeevolving environments. We also have proven analytically that the subconscious pathway is robust against uncertainty in the sensory information.

The cognitive skills exhibited by our robot mimic navigation abilities observed in animals and humans. On the one hand the robot is able to understand consciously novel (either dynamic or static) environments and decide on the best way to reach a target or even to conclude whether the situation has a solution or not. On the other hand the robot learns and memorizes successful (in terms of safe target reaching) experiences. Then if it faces a familiar situation (need not to be exactly the same), the subconscious pathway quickly retrieves the previously found successful solution. This functional flexibility provides the robot with versatility critical for efficient navigation in realistic complex environments. Our results also show that internal representation of the external world is subjective. The subconscious pathway transforms the sensory information in a non-orthogonal way. Then according to our experience we may perceive different situations as similar while similar situations as different. Such "confusions" are widely known in many psychological experiments.

\section{A. CIR as a core for cognitive navigation. Global vs local strategies}

Traditional cognitive maps implicitly rely on the knowledge of spatial structure of the environment. If the environment changes, the agent must adjust the map. Therefore the adaptation of spatial relationships is local in time [28]. Such flexibility is far from the global anticipating behavior observed in animals and humans [35]. The CIR theory revisits the concept of cognitive maps and offers global perspective for description of dynamic situations.

The most challenging issue is how to code time-evolving situations and agent's reactions in a neural structure. CIR offers an elegant solution to this problem [41]. On the one hand CIR generalizes spatial relationships by constructing static objects from spatiotemporal elements in the environment. On the other hand traditional cognitive maps are goal-independent [16]. However, different goals (targets) can coexist and CIR provides the agent with the required goal-flexible information.

Thus the CIR theory implicitly introduces an operational definition for global cognitive navigation in dynamic environments based on generalized cognitive maps. Such maps permit the agent to understand the situation in terms of identification of dangerous and desirable spatiotemporal regions.

\section{B. Architecture of the cognitive neural network}

The proposed neural network architecture has been inspired by the functional structure of evolved mammalian brain. Experimental insights show that processing of novel and familiar tasks involves different brain areas [51], [52]. When a task is successfully performed the obtained skill is refined and memorized by the subject.

In a similar way our neural network includes two different pathways linked in a closed loop through a block testing the solution success. If the robot is involved in a novel situation and the subconscious pathway cannot provide an effective solution, then the conscious pathway, made of Trajectory Modeling and Causal Neural Networks, generates new CIR. This process involves relatively long-lasting computations as it also happens with subjects solving novel tasks. Then successful solutions pass to the subconscious pathway, where an associative long term memory learns them. This is facilitated by the fact that CIRs are static cognitive maps, i.e. constant matrices or vectors. 


\section{Experimental assessment}

Our experimental setup reproduces the essence of prototypic situations observed in Nature: a cognitive agent pursues a moving target and avoids moving obstacles. Nevertheless we made several assumptions.

1. The robot moves in the arena with constant velocity. This restriction, however, is not a strong pitfall of the concept. Indeed, living beings usually run with constant velocity (slow, moderate or fast) optimal from e.g. the energy consumption viewpoint. Strong alteration may happen when the agent accidentally realizes that the selected strategy must be corrected. Such changes are local in time and space and can be implemented through one of the local navigation rules already existing in the literature.

2. The CIR concept provides global solutions, i.e. the robot can detect situations with no path to the target (for examples see [41], [45]) or solution may be too dangerous. Then the robot could wait until the situation changes. This, however, adds unnecessary complexity to the setup. Thus we designed experiments in such a way that an acceptable solution always exists and the robot immediately starts moving to the target.

3. The experiments here presented concern indoor navigation, i.e. light conditions, perspective of the visual acquisition, and camera vibrations are controlled. Outdoor navigation introduces uncertainty into the visual information. Then perceived situation may not match with the previously learned. To account for this possibility we have shown theoretically that the approach is robust against sensory noise.

\section{Performance of the approach}

In this work we have tested several critical parts of the robot's architecture. We have shown that:

1. The neural network responsible for predicting trajectories reaches high fidelity (about 99.9\%) in less than 50 training cycles. We note that this network is universal, i.e. once it has been trained there is no need for further adjustment.

2. CIRs provided by the conscious pathway faithfully describe dynamic situations and the robot always catches a target and avoids obstacles. On its path to the target the robot may deviate from the given trajectory by $2.6 \%$ ( $\mathrm{std}=0.18 \%$, $n=4$ ). The mean shortest distance to the obstacles and to the target is about $150 \%$ and $30 \%$ of the agent's size, respectively, which is acceptable for most applications.

3. The subconscious pathway requires about 200 offline training cycles to learn completely three similar but different dynamic situations.

4. On a standard PC (Matlab R2011a) the conscious and subconscious pathways provide CIR in about $27 \mathrm{~s}$ and 0.4 $\mathrm{s}$, respectively. The latter time is of the order of human reaction. These numbers can be reduced by several orders of magnitude using hardware implementation. For example, FPGA implementation of the conscious pathway provides CIRs in less than 0.25 s, i.e. about 100 folds faster than PC [49]. We also note that the performance is independent on the number of obstacles and targets in the arena.

5. The subconscious pathway is robust against sensory noise. The noise impact grows no more than linearly with the noise level.

\section{E. Strengths of the novel architecture}

The proposed approach for cognitive navigation by design possesses several inherit strength points:

1. Generation of CIRs is universal. It takes the same time to generate CIR of an empty arena and CIR of a crowd dynamic situation.

2. Fast and efficient navigation. The proposed architecture simulates decision-making in living beings. Navigation in familiar environments is "subconscious", i.e. no heavy computation is required.

3. Offline learning of typical experiences. Similar to the memory consolidation during sleep, our agent learns successfully solved situations offline (no motor execution is required). It is possible since the learning is independent on the sequence of items to be learned. Moreover, robots have a potential advantage over living beings. They can be preprogramed, i.e. the memory matrix $W$ [Eq. (8)] can be initialized either with zeros (tabula rasa) or with certain values (experiences learned by other robots). Then such a preprogramed robot during its life will learn only novel scenarios.

4. Robustness against sensory noise. The architecture allows only linear impact of noise on the quality of CIRs. For moderate noise level the robot correctly retrieves CIRs from the memory and solves familiar situations.

\section{ACKNOWLEDGMENT}

This work has been supported by the former Spanish Ministry of Science and Innovation (grant FIS2010-20054) and by the Russian Ministry of Education and Science (contract 14.B37.21.1237).

\section{REFERENCES}

[1] B. Milner, L.R. Squire, and E.R. Kandel, "Cognitive neuroscience and the study of memory", Neuron, Vol. 20, 445-468, 1998.

[2] R. Menzel and U. Müller, "Learning and memory in honeybees: From behavior to neural substrates", Annual Review of Neuroscience, Vol. 19, 379-404, 1996.

[3] R.R. Llinas and S Roy, "The prediction imperative as the basis for selfawareness", Philosophical Transactions of the Royal Society B, Vol. 364, 1301-1307, 2009.

[4] R.A.P. Roche, M.A. Mangaoang, S. Commins, and S.M. O'Mara, "Hippocampal contributions to neurocognitive mapping in humans: A new model", Hippocampus, Vol. 15, 622-641, 2005.

[5] J. O'Keefe and J. Dostrovsky, “The hippocampus as a spatial map. Preliminary evidence from unit activity in the freely-moving rat", Brain Research, Vol. 34(1), 171-175, 1971.

[6] E.L. Moser and M.B. Moser, "A metric for space", Hippocampus, Vol. 18(12), 1142-1156, 2008

[7] J. McIntyre, M. Zago, A. Bertho, and F. Lacquaniti, "Does the brain model Newton's laws?", Nature Neuroscience, Vol. 4, 693-694, 2001.

[8] T.S. Collett and J. Zeil, "Places and landmarks: An arthropod perspective". In: Healy S (ed) Spatial representation in animals. Oxford University Press, Oxford, 18-53, 1998.

[9] E.L. Moser, E. Kropff, and M.B. Moser, "Place cells, grid cells, and the brain's spatial representation system", Annual Review of Neuroscience, Vol. 31, 69-89, 2008.

[10] T. Hafting, M. Fyhn, S. Molden, M.B. Moser, and E.L. Moser, "Microstructure of a spatial map in the entorhinal cortex", Nature, Vol. 436(7052), 801-806, 2005.

[11] P. Raja and S. Pugazhenthi, "Optimal path planning of mobile robots: A review", International Journal of Physical Sciences, Vol. 7(9), 1314$1320,2012$.

[12] O. Khatib, "Real time obstacle avoidance for manipulators and mobile robots", International Journal of Robotics Research, Vol. 5(1), 90-98, 1986. 
[13] C.I. Connolly, J.B. Burns, and R. Weiss, "Path Planning Using Laplace's Equation", Proceedings of the IEEE International Conference on Robotics and Automation, 2102-2106, 1990.

[14] G.K. Schmidt and K. Azarm, "Mobile robot navigation in a dynamic world using an unsteady diffusion equation strategy", Proceedings of International Conference on Intelligent Robots and Systems IEEE/RSJ, 642-647, 1992.

[15] C. Qixin, H. Yanwen, and Z. Jingliang, "An evolutionary artificial potential field algorithm for dynamic path planning of mobile robot", Proceedings of the International Conference on Intelligent Robots and Systems, 2006

[16] M.O. Franz and H.A. Mallot, "Biomimetic robot navigation", Robotics and Autonomous Systems, Vol. 30, 133-153, 2000

[17] H. Cruse, "A recurrent network for landmark-based navigation", Biological Cybernetics, Vol. 88(6), 425-437, 2003.

[18] P. Varona, M.I. Rabinovich, A.I. Selverston, and Y.I. Arshavsky, "Winnerless competition between sensory neurons generates chaos: A possible mechanism for molluscan hunting behavior", Chaos, Vol. 12, 672677, 2002.

[19] H. Cruse and R. Wehner, "No need for a cognitive map: Decentralized memory for insect navigation", PLoS Computational Biology, Vol. 7(3): doi:10.1371/journal.pcbi.1002009, 2011.

[20] V.A. Makarov, N.P. Castellanos, and M.G. Velarde, "Simple agents benefit only from simple brains", Transactions on Engineering, Computing and Technology, Vol. 15, 25-30, 2006.

[21] M. Vergassola, E. Villermaux, and B. Shraiman, "Infotaxis as a strategy for searching without gradients", Nature, Vol. 445, 406-409, 2007.

[22] J. O'Keefe and L. Nadel. The hippocampus as a cognitive map. Clarendon Press, 1978

[23] A.V. Samsonovich and G.A. Ascoli, "A simple neural network model of the hippocampus suggesting its pathfinding role in episodic memory retrieval", Learning and Memory, Vol. 12, 193-208, 2005.

[24] B. Schmidt and A.D. Redish, "Navigation with a cognitive map", Nature, Vol. 497, 42-43, 2013.

[25] H. Mallot, H. Bülthoff, P. Georg, B. Schölkopf, and K. Yasuhara, "Viewbased cognitive map learning by an autonomous robot", Proceedings of International Conference on Artificial Neural Networks, Vol. 95, 381386, 1995.

[26] M.J. Mataric, "Navigating with a rat brain: A neurobiologically-inspired model for robot spatial representation". In: J.A. Meyer, S.W. Wilson (Eds.), From Animals to Animats, MIT Press, Cambridge, MA, 1991.

[27] C. Urdiales, A. Bandera, E. Pérez, A. Poncela, and F. Sandoval, "Hierarchical planning in a mobile robot for map learning and navigation", Studies in Fuzziness and Soft Computing, Vol. 116, 165-188, 2003.

[28] W. Yan, C. Weber and S. Wermter, "A neural approach for robot navigation based on cognitive map learning", Proceedings of the International Joint Conference on Neural Networks, 2012.

[29] V. Kunchev, L. Jain, V. Ivancevic, and A. Finn, "Path planning and obstacle avoidance for autonomous mobile robots: A review", Lecture Notes in Computer Science, Vol. 4252, 537-544, 2006.

[30] P. Vadakkepat, K.C. Tan, and M.L. Wang, "Evolutionary artificial potential fields and their application in real time robot path planning", Proceedings of Congress on Evolutionary Computation, 256-263, 2000.

[31] R. Araújo, "Prune-Able fuzzy ART neural architecture for robot map learning and navigation in dynamic environments", IEEE Transactions on Neural Networks, Vol. 17(5), 1235-1249, 2006.

[32] U. Steinkühler and H. Cruse, "A holistic model for an internal representation to control the movement of a manipulator with redundant degrees of freedom", Biological Cybernetics, Vol. 79, 457-466, 1998.

[33] S.X. Yang and M.Q.-H. Meng, "Real-time collision-free motion planning of a mobile robot using a neural dynamics-based approach", IEEE Transactions on Neural Networks, Vol. 14(6), 1541-1552, 2003.

[34] D.V. Lebedev, J.J. Steil, and H.J. Ritter, "The dynamic wave expansion neural network model for robot motion planning in time-varying environments", Neural Networks, Vol. 18(3), 267-285, 2005.

[35] A. Riegler, "The role of anticipation in cognition", In: Dubois, D. M. (ed.) Computing Anticipatory Systems. Proceedings of the American Institute of Physics, 2001.

[36] A.Z. Nasrollahy and H.H.S. Javadi, "Using particle swarm optimization for robot path planning in dynamic environments with moving obstacles and target", Third European Symposium on Computer Modeling and Simulation, 60-65, 2009.

[37] Y. Wang, D. Mulvaney, and I. Sillitoe, "Genetic-based mobile robot path planning using vertex heuristics", Proceedings of IEEE International Conference on Cybernetics and Intelligent Systems, 463-468, 2006.
[38] M.A.P. Garcia, O. Montiel, O. Castillo, R. Sepulveda, and P. Melin, "Path planning for autonomous mobile robot navigation with ant colony optimization and fuzzy cost evaluation", Applied Soft Computing, Vol. 9, 1102-1110, 2009.

[39] P. Raja and S. Pugazhenthi, "Path planning for a mobile robot in dynamic environments", International Journal of the Physical Sciences, Vol. 6(20), 4721-4731, 2011.

[40] R.A. Conn and M. Kam, "Robot motion planning on N-dimensional star worlds among moving obstacles", IEEE Transactions on Robotics and Automation, Vol. 14(2), 320-325, 1998

[41] J.A. Villacorta-Atienza, M.G. Velarde, and V.A. Makarov, "Compact internal representation of dynamic situations: Neural network implementing the causality principle", Biological Cybernetics, Vol. 103, 285297, 2010.

[42] V.A. Makarov and J.A. Villacorta-Atienza, "Compact internal representation as a functional basis for protocognitive exploration of dynamic environments", in Recurrent Neural Networks for Temporal Data Processing. InTech. pp. 81-102, 2011.

[43] V.A. Makarov, Y. Song, M.G. Velarde, D. Hubner, and H. Cruse, "Elements for a general memory structure: properties of recurrent neural networks used to form situation models", Biological Cybernetics, Vol. 98, 371-395, 2008.

[44] T. Eiter and H. Mannila, "Computing discrete Fréchet distance", Tech. Report CD-TR 94/64, Christian Doppler Laboratory for Expert Systems, 1994.

[45] Videos with robot experiments and numerical simulations are available at http://www.mat.ucm.es/ vmakarov/research.php

[46] J. Nassour, V. Hugel, and F.B. Ouezdou, and G. Cheng, "Qualitative adaptive reward learning with success failure maps: Applied to humanoid robot walking", IEEE Transactions on Neural Networks and Learning Systems, Vol. 24(1), 81-93, 2013.

[47] S. Kühn, W.J. Beyn, and H. Crus, "Modelling memory functions with recurrent neural networks consisting of input compensation units: I. Static situations", Biological Cybernetics, Vol. 96, 455-470, 2007.

[48] S. Kühn, W.J. Beyn, and H. Crus, "Modelling memory functions with recurrent neural networks consisting of input compensation units: II. Dynamic situations", Biological Cybernetics, Vol. 96, 471-486, 2007.

[49] L. Salas-Paracuellos, L. Alba, J.A. Villacorta-Atienza, and, V.A. Makarov, "FPGA implementation of a modified FitzHugh-Nagumo neuron-based causal neural network for compact internal representation of dynamic environments", Proceedings of SPIE Microtechnologies in Bioelectronics, Biomedical, and Bio-inspired Systems, 80680J - 9, 2011.

[50] P. Mukhopadhyay, Multivariate Statistical Analysis. World Scientific, 2009.

[51] S.E. Petersen, H. Van Mier, J.A. Fiez, and M.E. Raichle, "The effects of practice on the functional anatomy of task performance", Proceedings of the National Academy of Sciences USA, Vol. 95, 853-860, 1998.

[52] J.M. Fuster, The Prefrontal Cortex. Elsevier, London, 2008. 